\title{
DES OF THE TURBULENT FLOW AROUND A CIRCULAR CYLINDER OF FINITE HEIGHT
}

\author{
A. H. Elbatran \\ Marine Engineering Department, College of Engineering and Technology, Arab Academy for Science and Technology and \\ Maritime Transport, 1029, Alexandria, Egypt, E-Mail: a.elbatran@aast.edu
}

\begin{abstract}
The current research work investigates numerically the turbulent flow field characteristics around three dimensional circular cylinder of finite height at Reynolds number of 43000 using Detached Eddy Simulation (DES) turbulence model. The numerical results have been compared with the experiment and theoretical results obtained by other researchers. The results reveal that the DES turbulence model is superior to LES for predicting the flow past the circular cylinder of finite height at this Reynolds number. The numerical results of the current study show the great potential of the presented DES for investigating the complicated flow structure in this case. DES is very accurate for predicting the flow characteristics in many sophisticated cases and can reduce the computational efforts during the simulation process in comparison with Large Eddy Simulation (LES) turbulence mathematical model.
\end{abstract}

Key words: Circular Cylinder, turbulence model, DES, LES.

\section{Introduction}

The flow around circular cylinder is one of the most important fundamental phenomena in fluid mechanics fields (Strelets, 2001; Zhang and Guo, 2006; Rostamy et al, 2012; Javadi and Kinai, 2014). Moreover, the flow over cylinder have a wide applications in engineering such as heat exchanger, nuclear cooling system, cooling towers, subsea pipelines and offshore platforms (Javadi and Kinai, 2014). Hence, vortex and recirculation flow was altered because of trailing vortices formed at free ends (Fröhlich and Rodi, 2004). Therefore, this kind of flow is very interesting to be studied from the engineering point of view especially the effects of the vortex shedding on the cylinder.

The simulation of this flow is a big challenge because the flow domain is complex and moving boundary should be used at this case (Bai et al, 2016). Nowadays, CFD is used to handle the flow over 2D or 3D cylinders for saving cost and time. Many researchers studied numerically the flow field characteristics around circular cylinder using different turbulence models (Javadi and Kinai, 2014, Fröhlich and Rodi, 2004, Bai et al, 2016, Frederich et al., 2008; Chen et al., 2015; Hu et al., 2015; Chung, 2015; Kumar et al., 2016; Zhang et al., 2016; Nguyen and Nguyen, 2016; Hsu et al., 2016; Alonzo et al., 2015; Kappler, 2002). However, most of them used large eddy simulation (LES) turbulence models in their study.

LES represents the most accurate results especially for high Reynolds numbers. It also predict accurately the flow near wall for turbulence structures. Then, Frohlich and Rodi (2004), Javadi and Kinai (2014), Zhang et al., (2016), and $\mathrm{Hu}$ et al., (2015) used LES to predict the flow characteristics around cylinder. Recently, many of researchers mainly depend on DES to predict flow over cylinders. DES reduced computational capabilities compared to LES however retaining much of the physical accuracy of the method. Consequently, computational results obtained by Frederich et al., (2008), Nguyen and Nguyen (2016), and Bai et al., (2016) showed that numerical simulations with DES model is in a good agreement with experimental studies.

The previous studies used DES to predict the flow past cylinder, however, the flow around cylinder of finite height is not studying yet using DES. Hence, the present study continues their works for investigating numerically the flow around circular cylinder of finite height using DES turbulence model of the finite volume CFD code ANSYS Fluent. In addition, in this study unstructured tetrahedral grid was used for the CFD solver to study the ability of such grid type on predicting the complex flow pattern in this case accurately. Comparison between the numerical results of the 
current study and the available experimental data had been made and very good agreement was found between the two results.

\section{Numerical Method and Modelling}

\subsection{Flow domain}

The present study of the flow around a circular cylinder of finite height corresponds to an experiment performed by Kappler (2002) in a water tunnel. The experiment conditions in this case were $R e=43000$, cylinder height to diameter ratio $H / D=2.5$, the boundary layer thickness of the approaching flow $\delta / H=0.1$ and the width of the tunnel $=7 \mathrm{D}$, the height of the tunnel $=5 \mathrm{D}$, the blockage of the tunnel $=7.3 \%$. The base of the cylinder located at the origin of the coordinate system and $x, y, z$, representing the streamwise, wall normal and lateral directions respectively as described in Fig. 1(a). The computational domain specifications are diameter of the cylinder $(D)=0.05 \mathrm{~m}$, height of the cylinder $(H)=2.5 \mathrm{D}$, the inlet boundary condition is located at $x / D=-7.5$, the outflow boundary condition is located at $x / D=12.5$ as shown in Fig. 1(b) and 1(c).

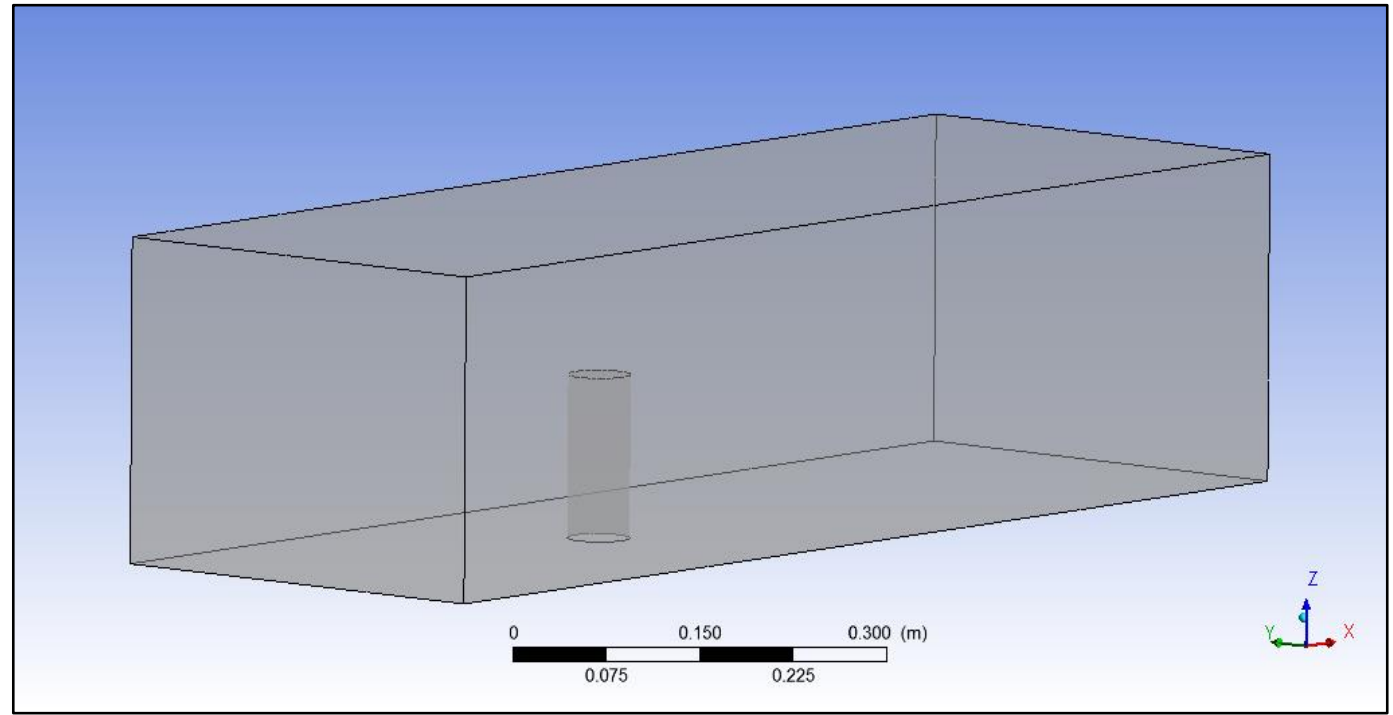

(a) Computational domain

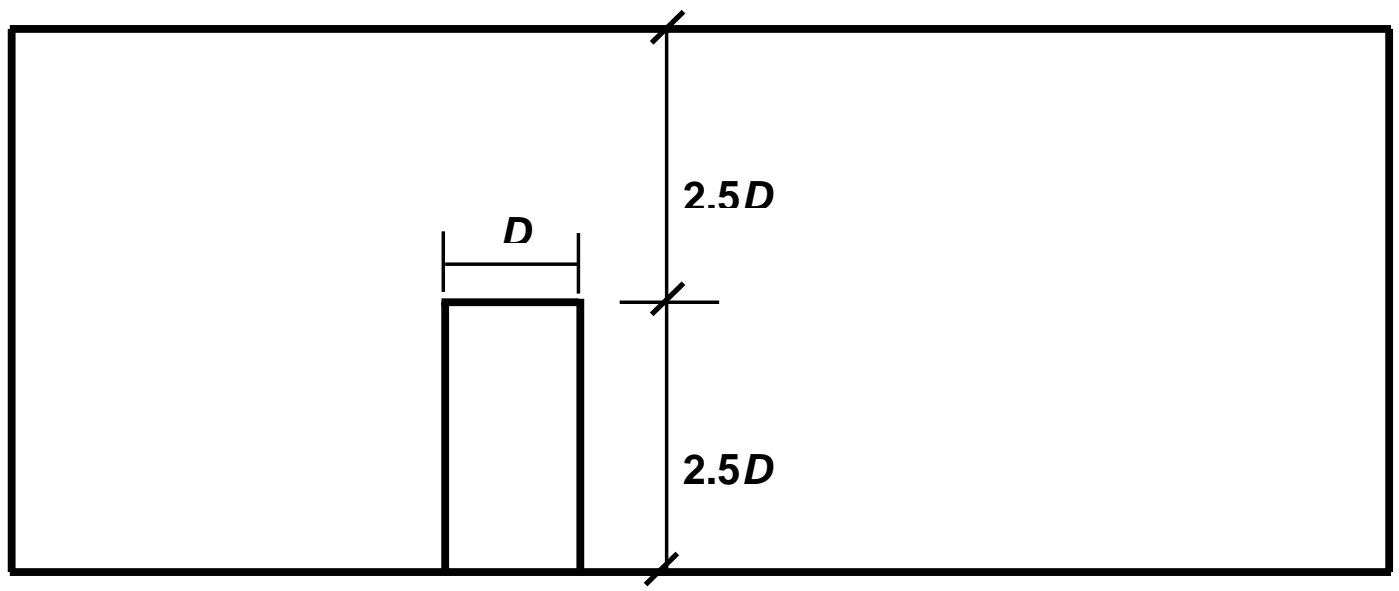

(b) Domain dimensions (side view) 


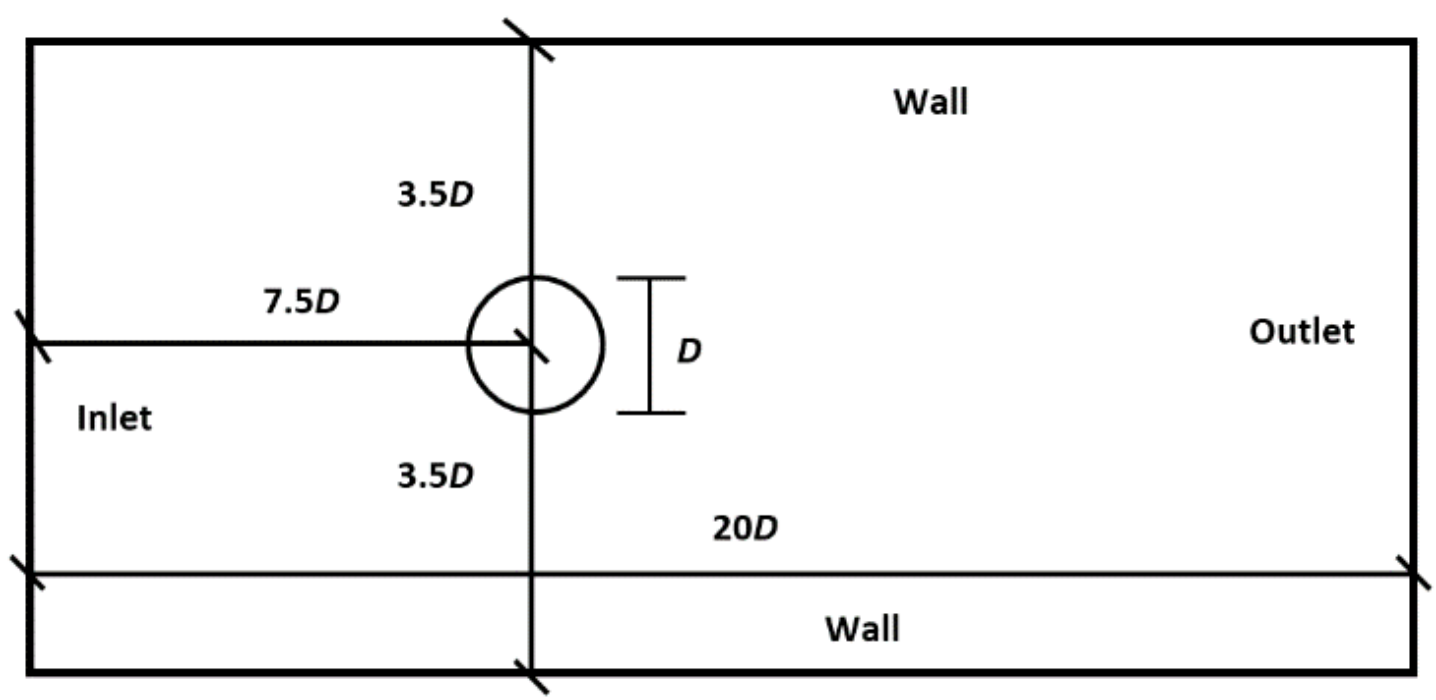

(c) Domain dimensions (plan view)

Fig. 1: Cylinder computational domain dimensions.

\subsection{Grid generation}

A grid generator ICEM CFD of the Reynolds-Averaged Navier-Stokes Equations (RANSE) code Ansys Fluent was used for meshing the system with unstructured tetrahedral grid as shown in Fig (2). The mesh elements were packed over the surface of the cylinder with 15 layers in the region of the boundary layer as illustrated in Fig. 3(c).

Four computational grids had been tested in this section study for determining the number of grid cells required to establish a grid-independent solution. The specifications of grid cells are described in Table 1. Fig. 3 shows the mean streamwise velocity determined numerically by the current DES turbulence models in $X$ direction which determined by four grids. It was observed that grid 3 and grid 4 gave acceptable and similar results while grid 4 required more time for solution. Hence, grid 3 was chosen to carry out the analysis of the current study.

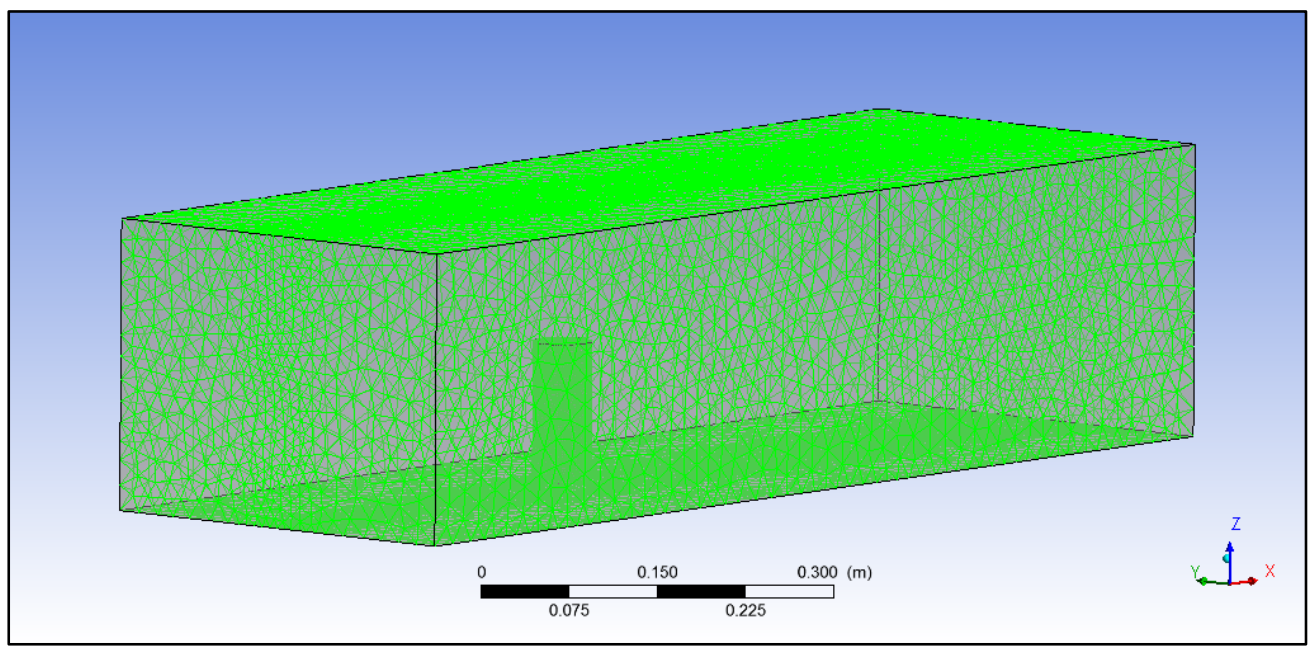

(a) All domain mesh view 


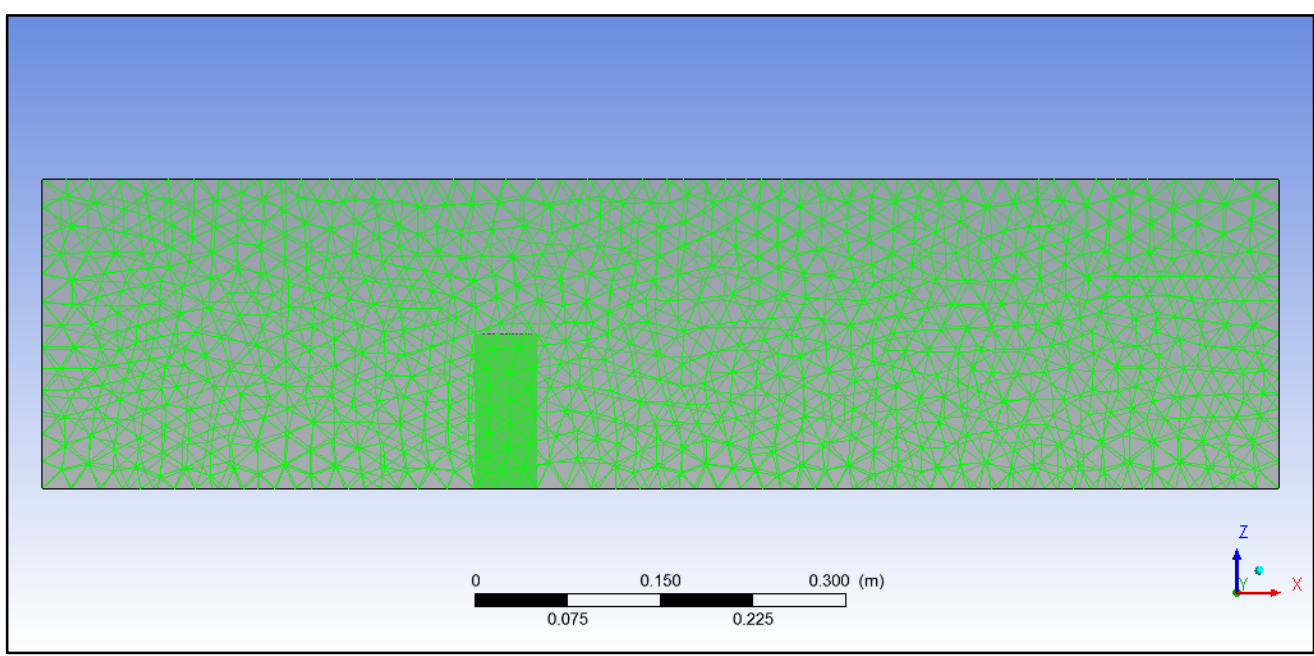

(b) Side view

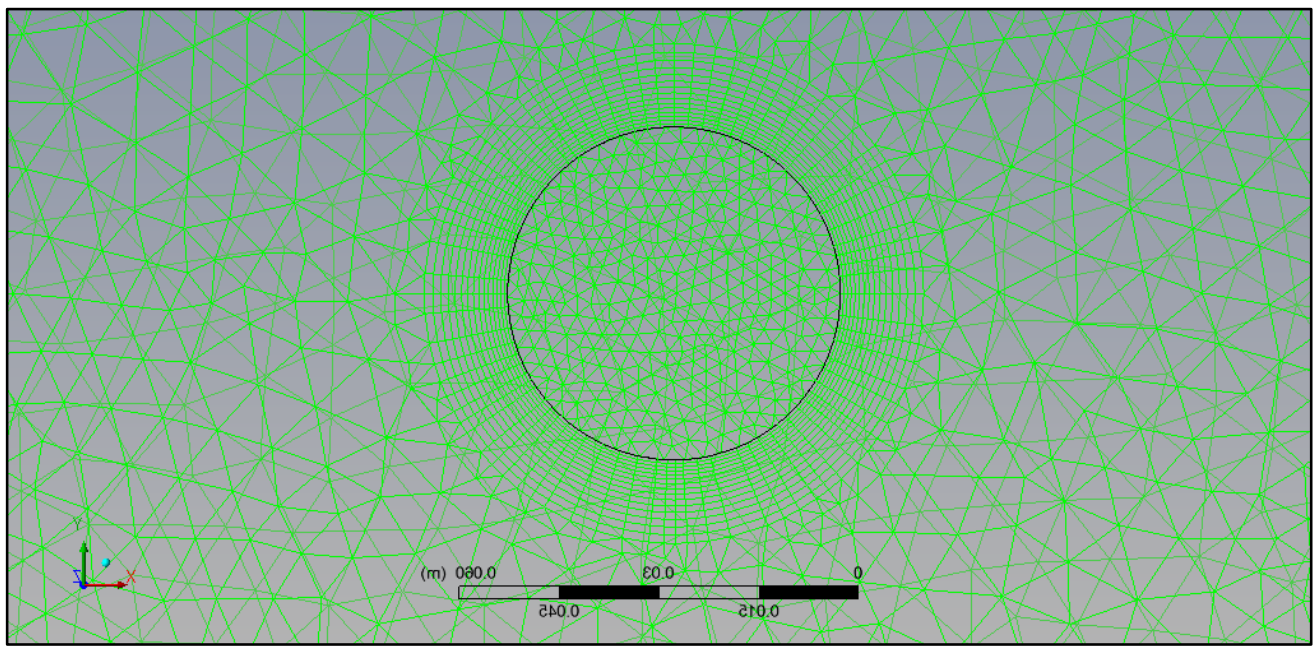

(c) Mesh plan view

Fig. 2: Computational domain with unstructured tetrahedral mesh elements.

Table 1 Grid specifications

\begin{tabular}{|c|c|}
\hline Grid & No. of cells \\
\hline 1 & 507114 \\
\hline 2 & 622587 \\
\hline 3 & 711696 \\
\hline 4 & 831225 \\
\hline
\end{tabular}

\subsection{Boundary conditions}

The circular cylinder surface was treated as a viscous wall with no slip boundary condition. The inflow boundary condition was set at the left end of the computational domain while the right hand side boundary was set as opening (the outflow boundary condition was used also and it give the same results as the opening boundary condition, but it cause some problems and affect the results in case of transient solution). The turbulence level at the inlet boundary condition was $5 \%$. The top and the sides of the computational domain were treated as symmetry plans. The bottom of the computational domain was taken as a wall of free slip. 


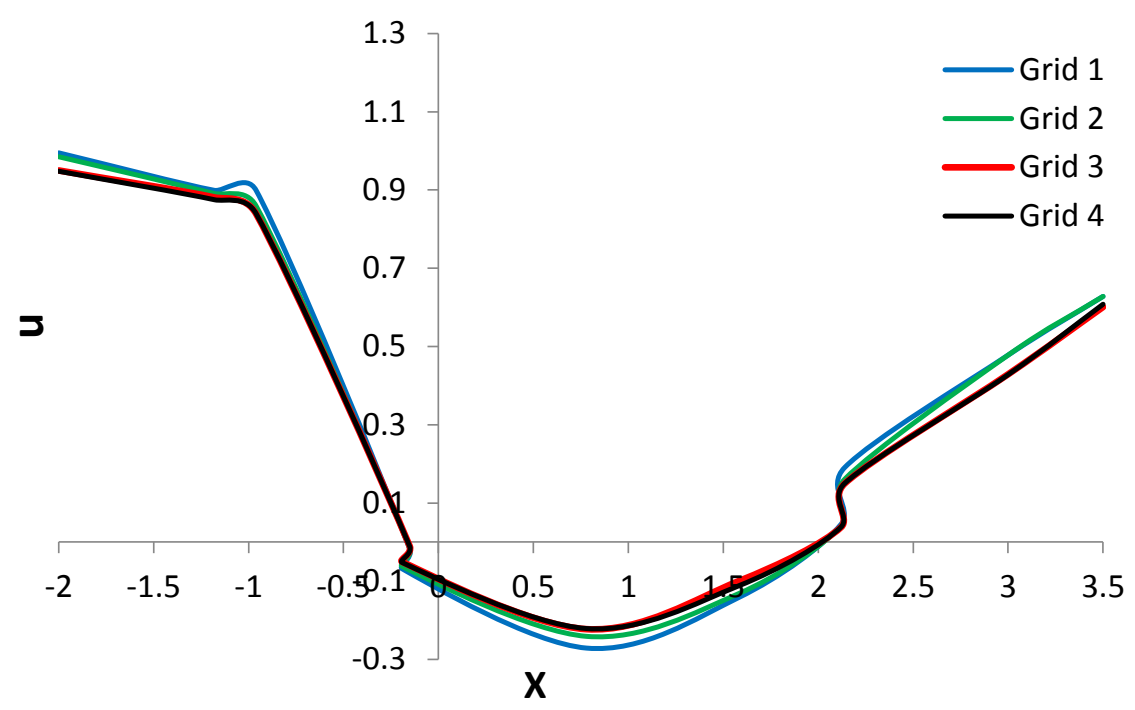

Fig. 3: Cylinder streamwise mean velocity distribution in $X$ direction at different grid.

\subsection{Computational method}

The velocity inlet and the pressure outlet boundary conditions were assigned to the inlet and outlet boundaries of the computational domain. The two sides of the 3D computational domain were defined as planes of symmetry at the different numerical simulations. The flow was considered as unsteady (transient) flow, the time step in all transient simulations was taken $0.001 \mathrm{sec}$, and the total time was 12 seconds. A second order upwind numerical scheme was used for discretizing the advection terms (Driver and Seegmiller, 1985). DES turbulence models have been used in this study to investigate its ability for predicting the complex flow characteristics for the given case. The solution convergence at the different simulations was monitored by dimensionless residual sum for all variables across the computational points and checking the drag coefficient of the circular cylinder. The residual absolute criteria were set to the value of $10^{-4}$ for checking the convergence of the solution.

\section{Results and Discussion}

The flow over circular cylinder with finite height is investigated numerically in this part. The numerical results obtained by using DES turbulence model are compared with experiment and other numerical turbulence model for the present case. Moreover, the flow characteristics are analyzed using current DES model.

\subsection{Model experiment and numerical comparisons}

This section discusses the impact of the current DES model on the results compared with experiment and LES model. Fig. 4 shows the mean streamwise velocity determined experimentally and numerically by LES and the current DES turbulence models in directions of $X$ and $Z$. The companion experiment is for Kappler (2002) where the LES model results are for Frohlich and Rodi (2004). It was noticed that the present DES turbulence model detected efficiently the mean streamwise velocity along and around the cylinder in comparison with experiment and LES numerical results. Although, the unstructured grid has been used in the current study, the model gave a confident results which are closer to the experimental data. This can save the time and computational efforts in comparison with the use of other turbulence models especially LES mathematical turbulence model with structured mesh. 


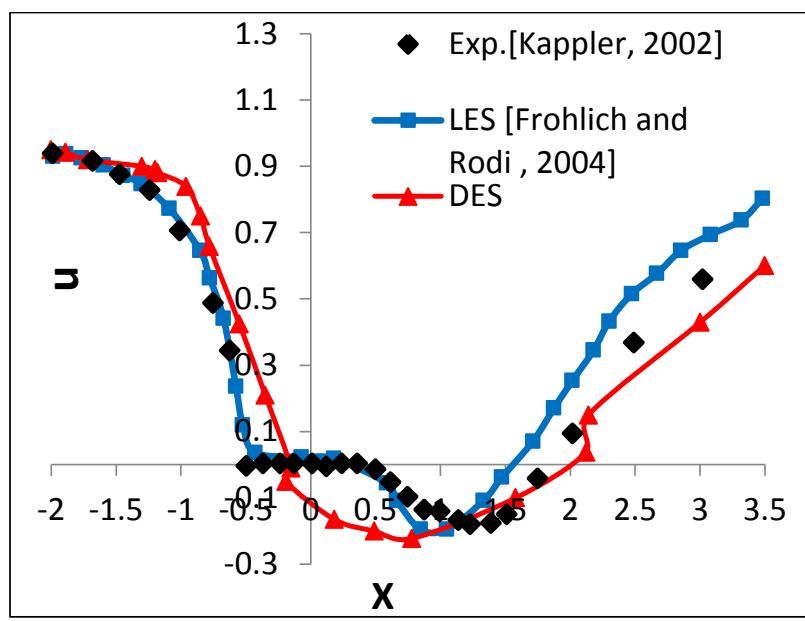

(a) $(Y / D=1.5, Z=0)$

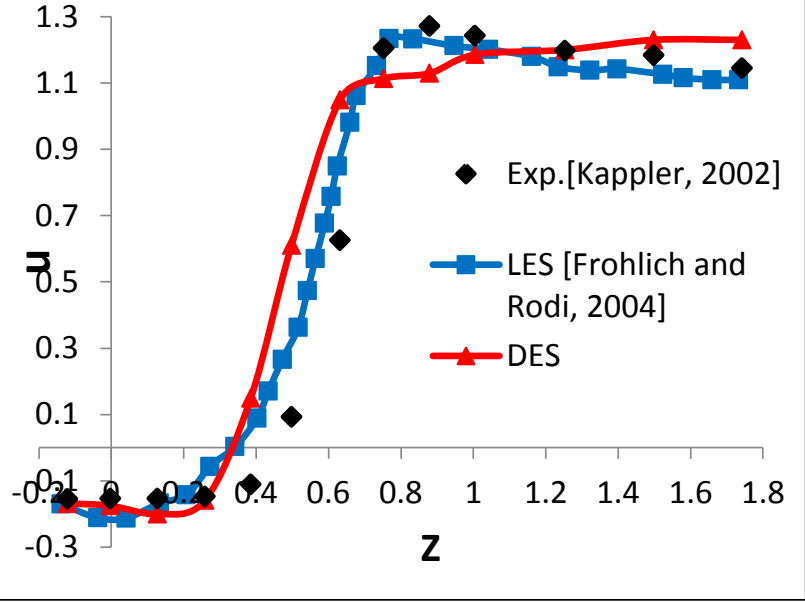

(b) $(Y / D=1.5, x=1)$

Fig. 4: Cylinder streamwise mean velocity distribution in $X$ and $Z$ directions

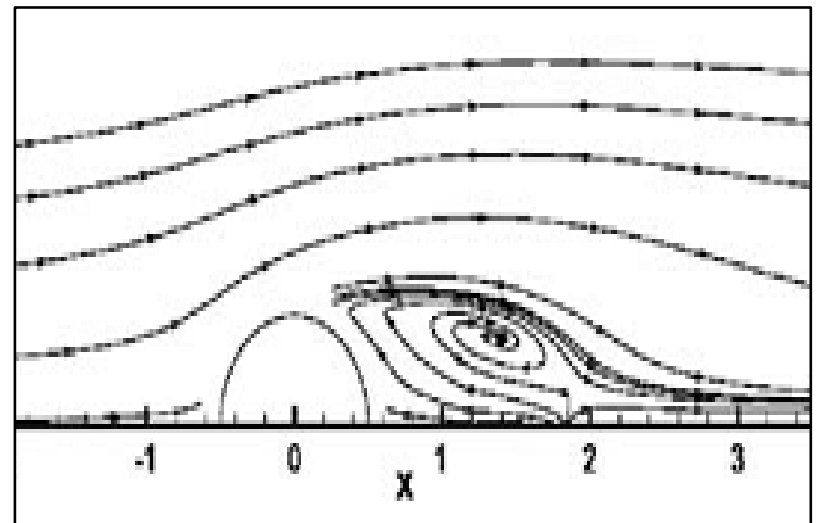

(a) Experimental results (Kappler, 2002)

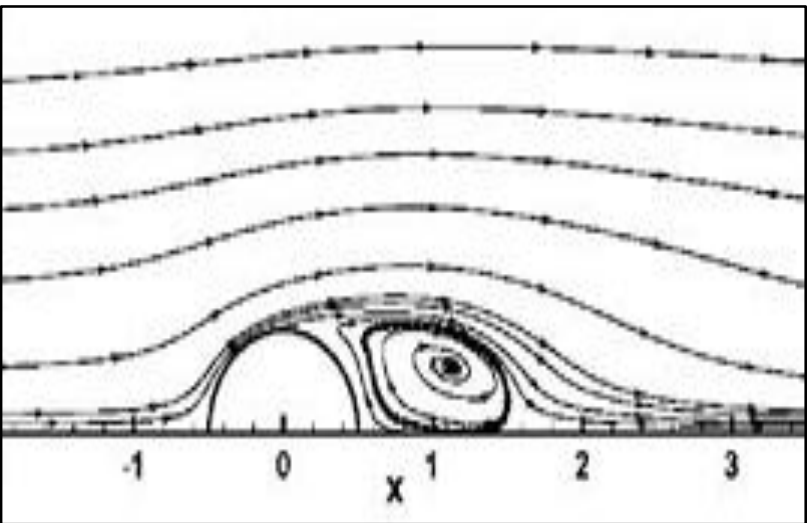

(b) LES mathematical turbulence model results (Frohlich and Rodi 2004)

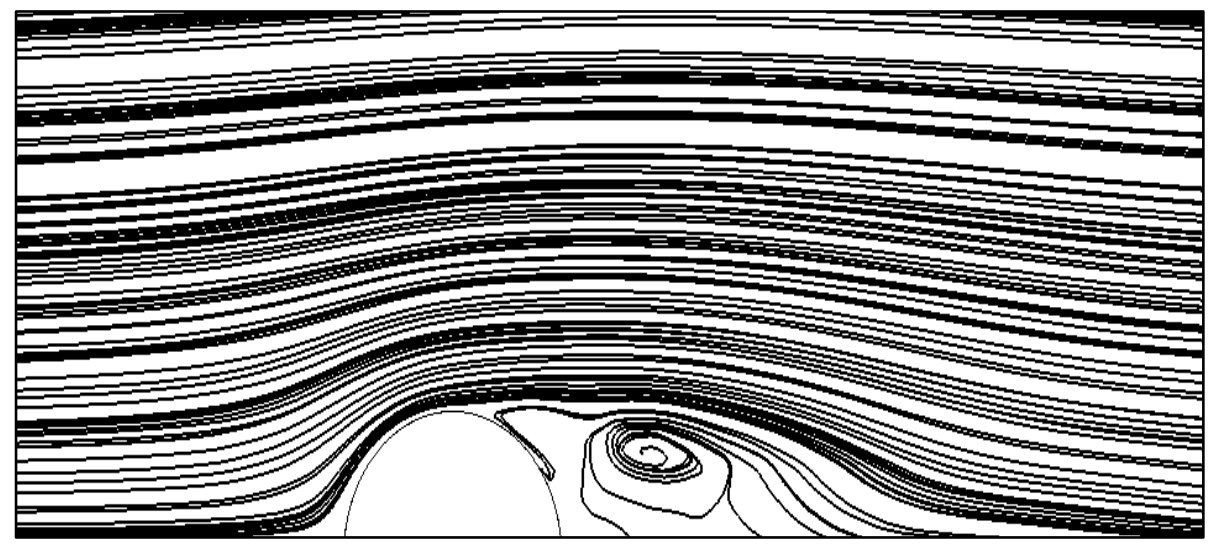

(c) DES turbulence model results

Fig. 5: Plan view for the streamlines over the cylinder of finite height

The streamlines of the flow around cylinder of finite height for the present DES turbulence model in comparison with experiment and numerical LES model results are showed in Figs. 5 and 6. The DES can detect sufficiently the flow over the cylinder of finite height especially the vortices formed behind the cylinder. Good agreement between the current numerical results of this study and experiment, which can be noticed in the streamlines of the flow. 


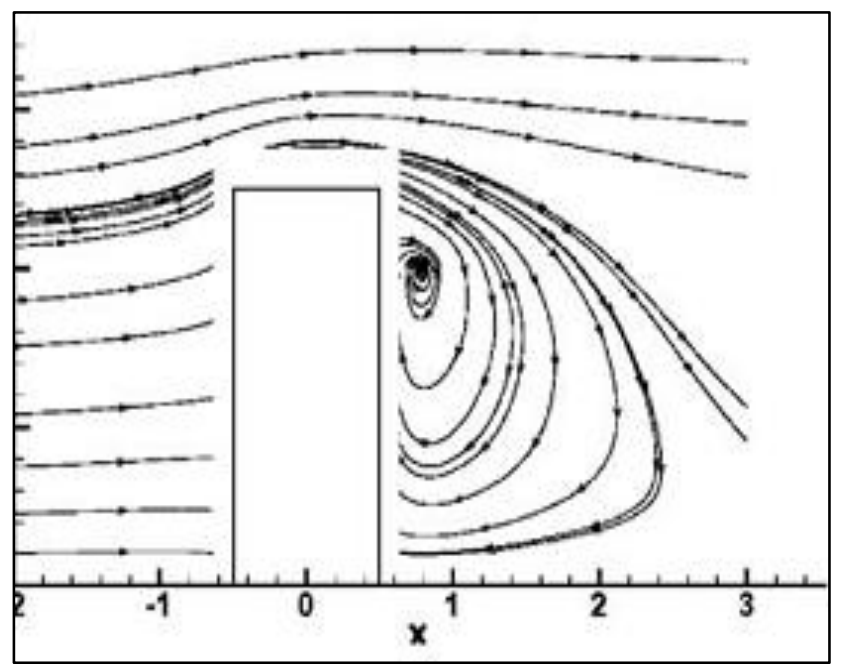

(a) Experimental results (Kappler, 2002)

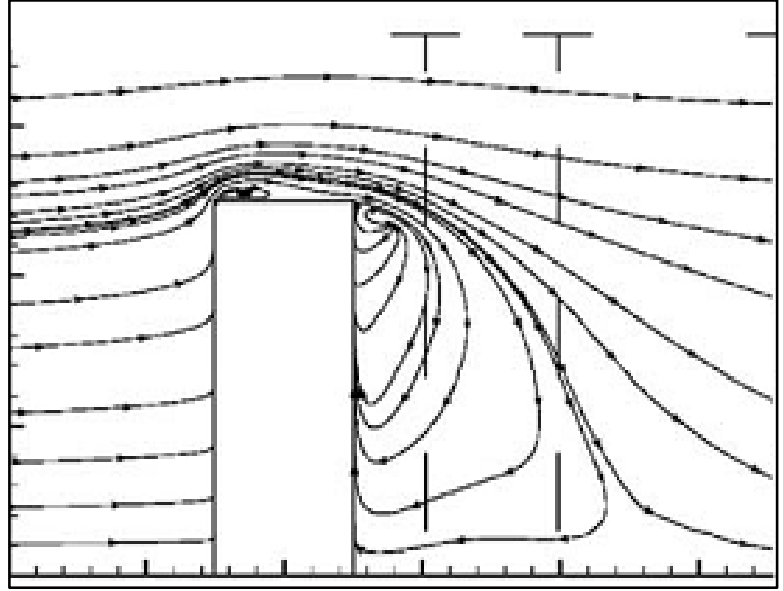

(b) LES mathematical turbulence model results (Frohlich and Rodi 2004)

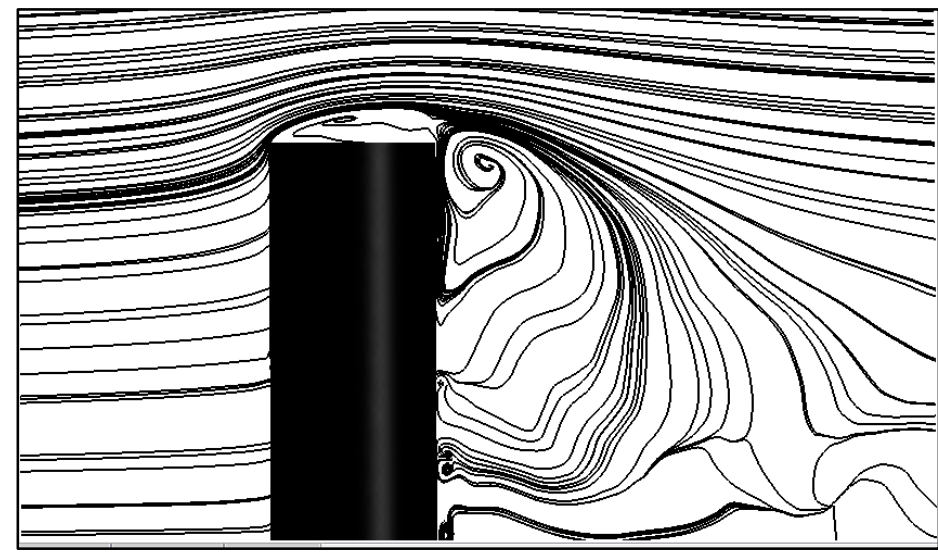

(c) DES turbulence model results

Fig. 6: Side view for the streamlines over the cylinder of finite height.

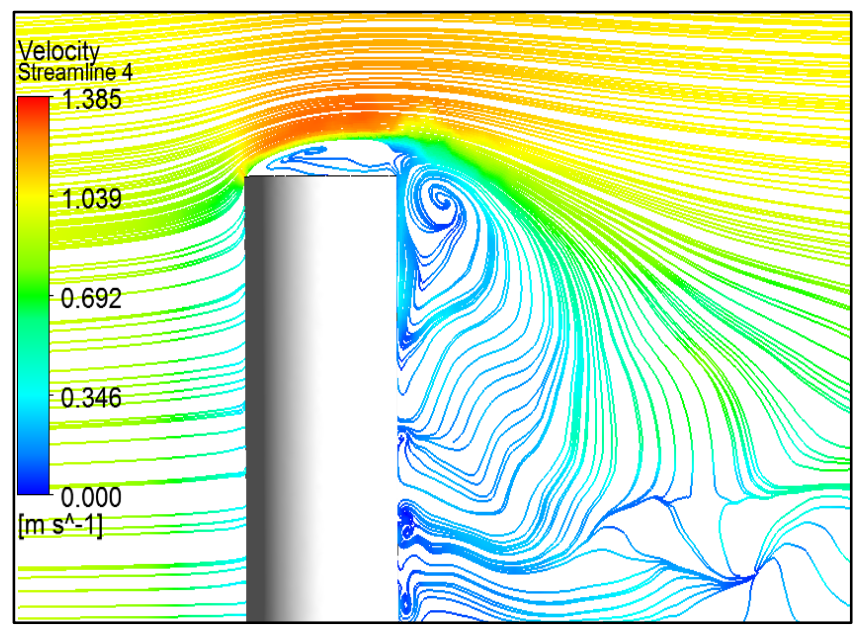

(a) Side view

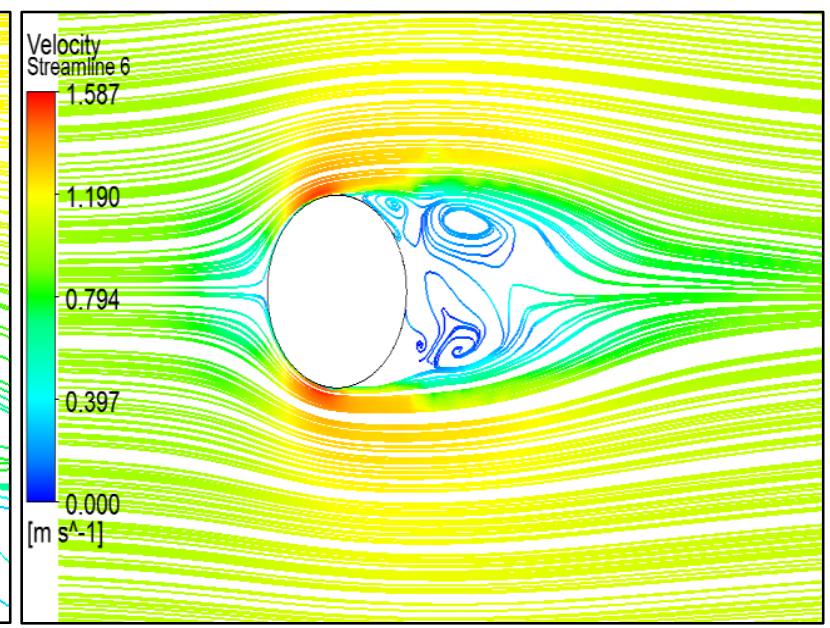

(b) Plan view

Fig. 7: Streamlines colored by velocity contours for DES model numerical results in two planes. 


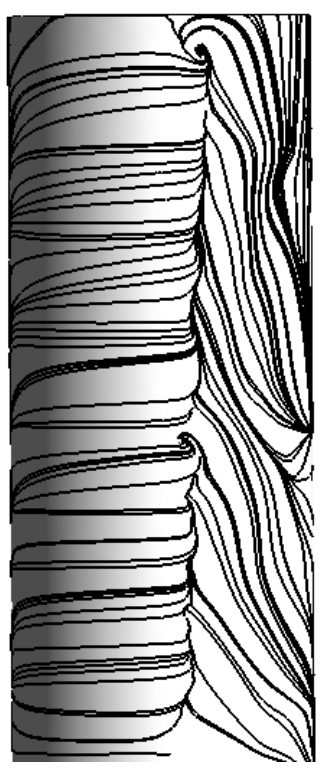

Fig. 8: Cylinder surface streamlines

\subsection{Numerical results}

Fig. 7 shows streamlines colored by flow velocity of the current DES turbulence model results around the circular cylinder of finite height. Fig. 8 illustrates the surface streamlines on the cylinder. From the previous figures, it can be seen that, the flow is highly complex and vortices are formed in the region behind the cylinder with pronounced separation in the cylinder wake region. In addition, the vortex shedding pattern concentrated at the tip of the cylinder.

Velocity vectors in the internal flow field over the circular cylinder in two planes are shown in Fig. 9. It was observed that the flow velocity was decelerated in the front when passing the cylinder, and then, the velocity of the flow was accelerated at the cylinder tips, producing jet flow. Later the velocity began to decrease behind the cylinder, where recirculation area can be formed.

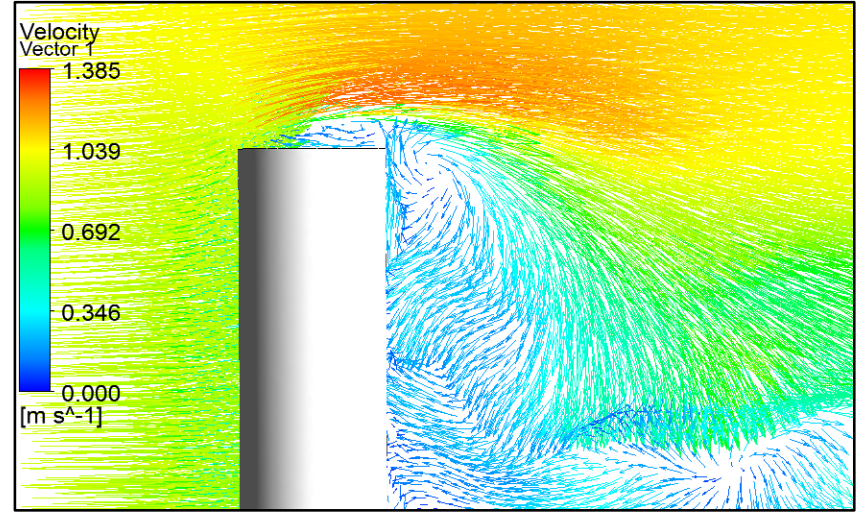

(a) Side view

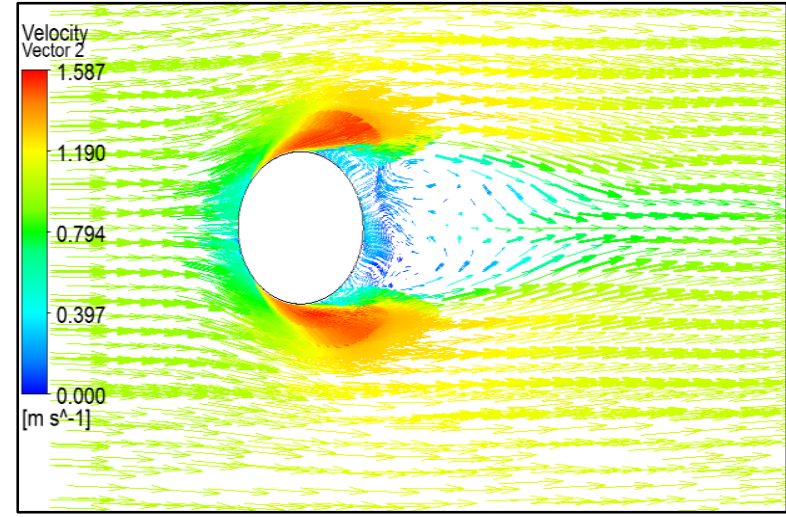

(b) Plan view

Fig. 9: Velocity vectors showing the flow pattern over the circular cylinder of finite height at two planes.

The flow around circular cylinder is complex flow case, therefore, turbulence measurement is an important factor to be considered when studying the flow pattern in the current case. Turbulence kinetic energy (TKE) is one of the most important formulas for measuring turbulence which expressed about the velocity variations with respect to time. Hence, Fig. 10 displays the contours of turbulence kinetic energy. The results indicate that the turbulence 
kinetic energy increased behind the cylinder and along downstream. Furthermore, the area at the upper edge of the cylinder becomes more highly turbulent due to the formation of the vortices at this region.

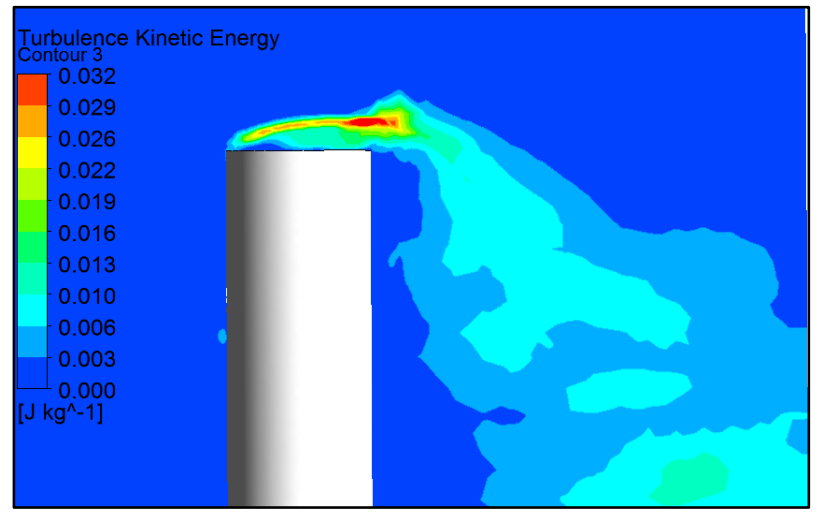

(a) Side view

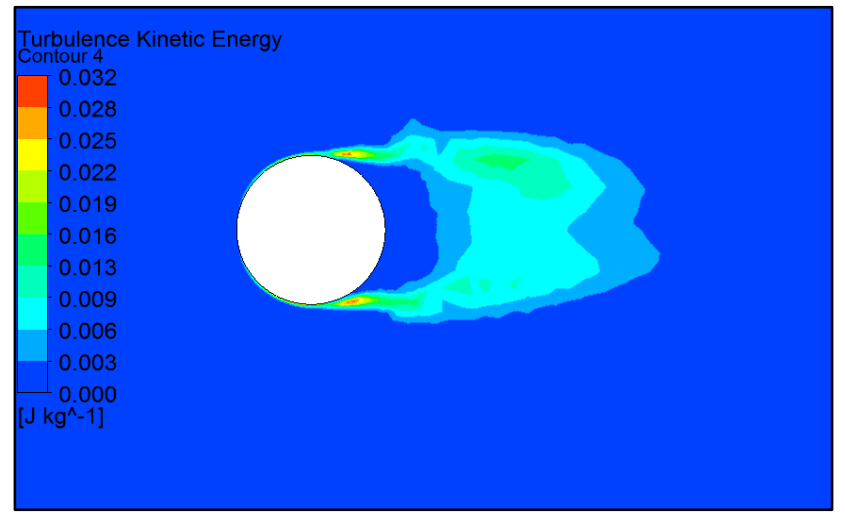

(b) Plan view

Fig. 10: Turbulence kinetic energy (TKE) contours in two planes.

Fig. 11 gives the drag coefficient $C_{D}=F_{x} / 0.5 \rho u_{\infty}^{2} H D$ with the simulated time which can be considered as a first criterion for assessment of the drag force $\left(F_{x}\right)$ of the circular cylinder in $x$ direction. The average drag coefficient obtained in the current study is 0.67 . It is closer to the experimental date was determined by Kawamura et al., (1984) and Okamoto and Sunabashiri (1992) where drag coefficients $C_{D}$ were 0.78 and 0.73 respectively in these studies.

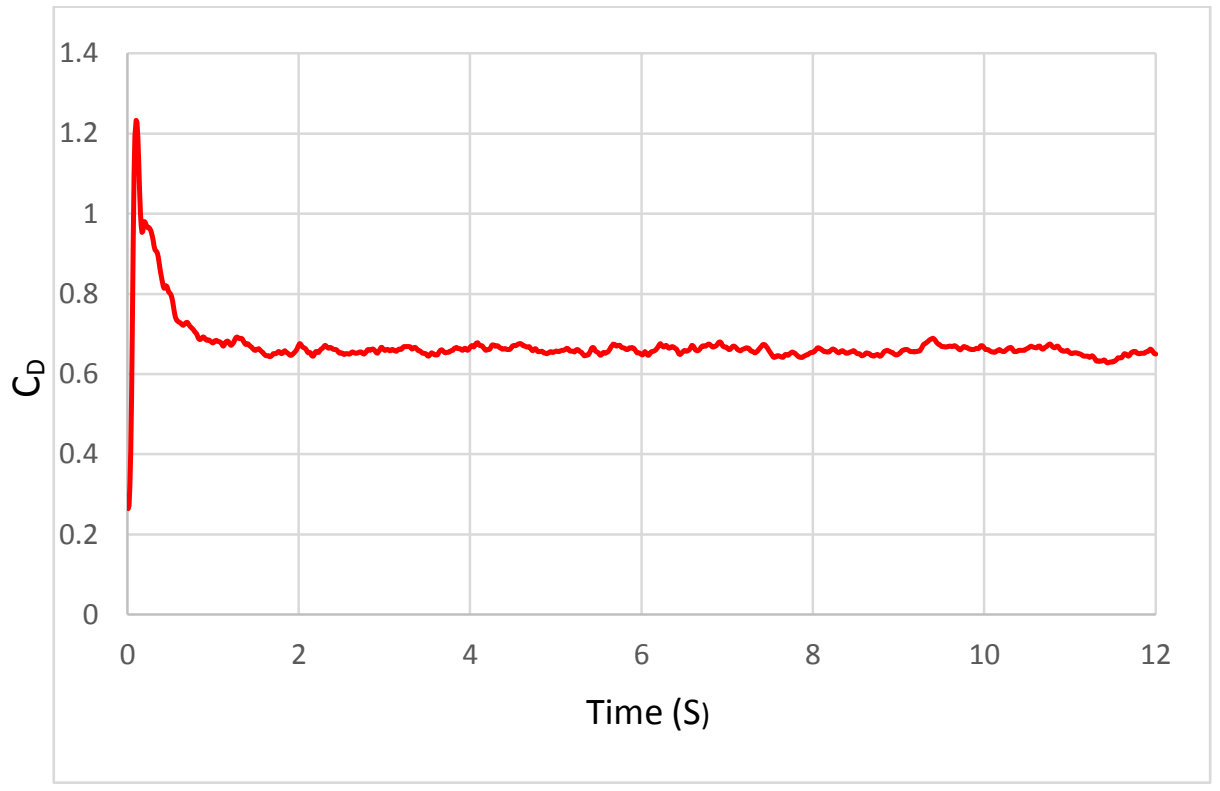

Fig. 11: Drag coefficient $C_{D}$ for the circular cylinder with finite height using DES turbulence model

\section{Conclusion}

DES turbulence model with unstructured tetrahedral grid have been presented in this study to investigate the flow field characteristics around circular cylinder of finite height. Comparison to experiment and numerical LES model proved that the results obtained with the present DES predicted efficiently the main characteristics of the complex flow around the cylinder of finite height. The results showed that the vortices, recirculation and massive separations are formed in the wake region behind the cylinder. The results gave acceptable value for the drag coefficient in comparison with experimental results. 


\section{Acknowledgement.}

The author would like to express his sincere gratitude to Arab Academy for Science and Technology and Maritime Transport, Egypt for its great help and support in this research work.

\section{References}

Alonzo-García, A., Gutierrez-Torres, C. D. C., Bernal, J. J., de Mollinedo-Ponce, L. H., Martinez-Delgadillo, S. A., and Barbosa-Salda-a, J. G. (2015): RANS simulations of the U and V grooves effect in the subcritical flow over four rotated circular cylinders, Journal of Hydrodynamics, Ser. B, vol. 27, No. 4, p. 569-578. https://doi.org/10.1016/S1001-6058(15)60518-2

Bai, W., Mingham, C. G., Causon, D. M., and Qian, L. (2016): Detached eddy simulation of turbulent flow around square and circular cylinders on Cartesian cut cells, Ocean Engineering, vol. 117, pp. 1-14. https://doi.org/10.1016/j.oceaneng.2016.03.009

Chen, W. L., Cao, Y., Li, H., and Hu, H. (2015): Numerical investigation of steady suction control of flow around a circular cylinder, Journal of Fluids and Structures, vol. 59, pp. 22-36. https://doi.org/10.1016/j.jfluidstructs.2015.09.002

Chung, M. H. (2015): Hydrodynamics of flow over a transversely oscillating circular cylinder beneath a free surface. Journal of Fluids and Structures, vol. 54, pp. 27-73. https://doi.org/10.1016/j.jfluidstructs.2014.10.006

Driver, D. M. and Seegmiller, H. L. (1985): Features of a Reattaching Turbulent Shear Layer in Divergent Channel Flow, AIAA Journal, vol. 23, No. 2, pp. 163-171. https://doi.org/10.2514/3.8890

Frederich, O., Wassen, E., and Thiele, F. (2008): Prediction of the flow around a short wall-mounted finite cylinder using LES and DES, JNAIAM, vol. 3(3-4), pp. 231-247.

Fröhlich, J. and Rodi, W. (2004): LES of the flow around a circular cylinder of finite height, International Journal of heat and fluid flow, vol. 25, No. 3, pp. 537-548. https://doi.org/10.1016/j.ijheatfluidflow.2004.02.006

Hsu, C. M., Huang, R. F., and Chung, H. C. (2016): Flow characteristics and drag force of a square cylinder in crossflow modulated by a slot jet injected from upstream surface, Experimental Thermal and Fluid Science, vol. 75, pp. 235-248. https://doi.org/10.1016/j.expthermflusci.2016.02.014

Hu, G., Tse, K. T., Kwok, K. C. S., and Zhang, Y. (2015): Large eddy simulation of flow around an inclined finite square cylinder, Journal of Wind Engineering and Industrial Aerodynamics, vol. 146, pp. 172-184. https://doi.org/10.1016/j.jweia.2015.08.008

Javadi, K., and Kinai, F (2014): On the turbulent flow structures over a short finite cylinder: numerical investigation, Proceedings of the International Conference on Heat Transfer and Fluid Flow Prague, Czech Republic, August 1112, 2014, Paper No. 129.

Kappler, M., (2002): Experimentelle Untersuchung der Umstromung evon Kreiszylindern mit ausgepren agt dreidimensionalen Effekten, Ph.D. thesis, Institute for Hydromechanics, University of Karlsruhe.

Kawamura, T., Hiwada, M., Hibino, T., Mabuchi, I., Kamuda, M (1984): Flow around a finite circular cylinder on a flat plate, Bull. JSME Vol. 27 (232), pp. 2142-2151. https://doi.org/10.1299/jsme1958.27.2142

Kumar, P. S., Vendhan, C. P., and Krishnankutty, P. (2016): Study of water wave diffraction around cylinders using a finite-element model of fully nonlinear potential flow theory, Ships and Offshore Structures, pp. 1-14.

Nguyen, V. T., and Nguyen, H. H. (2016): Detached eddy simulations of flow induced vibrations of circular cylinders at high Reynolds numbers, Journal of Fluids and Structures, vol. 63, pp. 103-119. https://doi.org/10.1016/j.jfluidstructs.2016.02.004

Okamoto, S., Sunabashiri, Y., (1992): Vortex shedding from a circular cylinder of finite length placed on a ground plane, J. Fluids Eng. Vol. 114, pp. 512-521. https://doi.org/10.1115/1.2910062

Rostamy, N., Sumner, D., Bergstrom, D. J., and Bugg, J. D. (2012): Local flow field of a surface-mounted finite circular cylinder. Journal of Fluids and Structures, 34, 105-122. https://doi.org/10.1016/j.jfluidstructs.2012.04.014

Strelets, M. (2001): Detached eddy simulation of massively separated flows, In AIAA, Aerospace Sciences Meeting and Exhibit, 39 th, Reno, NV. https://doi.org/10.2514/6.2001-879

Zhang, J., and Guo, Y. (2006): Large eddy simulation of 3D turbulent flow around deep-sea marina structure, ECCOMAS CFD 2006: Proceedings of the European Conference on Computational Fluid Dynamics, Egmond aan Zee, The Netherlands, September 5-8, 2006, Delft University of Technology; European Community on Computational Methods in Applied Sciences (ECCOMAS).

Zhang, K., Katsuchi, H., Zhou, D., Yamada, H., and Han, Z. (2016): Numerical study on the effect of shape modification to the flow around circular cylinders, Journal of Wind Engineering and Industrial Aerodynamics, vol. 152, pp. 23-40. https://doi.org/10.1016/j.jweia.2016.02.008 